\title{
AFM engine with optical actuation and readout printed on the facet of a multi-core fiber
}

\author{
Mareike Trappen $^{1,2}$, Philipp-Immanuel Dietrich ${ }^{1,2,3}$, Pascal Burger ${ }^{1,2}$, Matthias Blaicher ${ }^{1,2}$, Gerald Göring ${ }^{4,5}$, \\ Thomas Schimmel $^{4,5}$, Wolfgang Freude ${ }^{1}$, Hendrik Hölscher ${ }^{2}$ und Christian Koos ${ }^{1,2,3}$ \\ ${ }^{1}$ Institute of Photonics and Quantum Electronics (IPQ), Karlsruhe Institute of Technology (KIT), 76131 Karlsruhe, Germany \\ ${ }^{2}$ Institute for Microstructure Technology (IMT), Karlsruhe Institute of Technology (KIT), 76344 Eggenstein Leopoldshafen, Germany \\ ${ }^{3}$ Vanguard Photonics GmbH, Gablonzer Str. 10, 76185 Karlsruhe, Germany \\ ${ }^{4}$ Institute of Applied Physics (APH), Karlsruhe Institute of Technology (KIT), 76131 Karlsruhe, Germany \\ ${ }^{5}$ Institute of Nanotechnology (INT), Karlsruhe Institute of Technology (KIT), 76344 Eggenstein-Leopoldshafen, Germany \\ Author e-mail address: mareike.trappen@kit.edu, christian.koos@kit.edu
}

Abstract: Using two-photon lithography, we fabricate an ultra-compact atomic force microscope engine on the facet of a multi-core fiber. The AFM is optically actuated and read out, and it offers atomic step-height resolution in difficult-to-access areas. (1) 2020 The Author(s)

\section{Introduction}

Atomic force microscopy (AFM) [1] is a widely used tool for surface characterization and nanofabrication with highest resolution. The technique exploits the interaction of a mechanical probe with a sample surface. Conventional AFM implementations are realized by micro-machined silicon cantilevers equipped with sharp tips [1]. For actuation and read-out, these cantilevers must be mounted and aligned to a macroscopic opto-mechanical system, which essentially limits the application range to the investigation of predominantly planar surfaces. Bulky and alignmentsensitive read-out optics can be avoided by carving the cantilever out of a fused-silica single-mode fiber using focused ion-beam (FIB) milling, and by exploiting the optical back-reflection from the cantilever into the fiber core for position read-out [2]. This approach was shown to provide sub-nm step-height resolution, but its practical applicability is constrained by the complexity and the limited throughput of the underlying fabrication process. Moreover, the concept does not allow for an actuation of the cantilever alone, and therefore the AFM measurements could be performed in contact mode only. These limitations can be overcome by 3D-printed AFM engines, realized by multiphoton laser lithography on the facets of optical fiber arrays [3]. These AFM engines do not need any manual alignment and can be actuated and read out optically through glass fibers. However, while 3D-printed AFM engines offer unprecedented flexibility, the structures demonstrated so far are still built on comparatively large fiber arrays with typical lateral dimensions of several millimeters. This prevents operation in difficult-to-access areas such as drill holes, high aspect-ratio trenches, or cavities.

In this paper, we demonstrate ultra-compact 3D-printed AFM engines that are realized on the facets of $125 \mu \mathrm{m}$ thick multicore fibers (MCF). The AFM engines are optically actuated and read out through two of the seven singlemode fiber cores, thereby permitting fully remote operation. In our proof-of-principle experiments, we perform dynamic-mode measurements of steps on highly oriented pyrolytic graphite, demonstrating atomic step-height resolution. We further measure the surface of a ledge inside a laser-drilled hole with $250 \mu \mathrm{m}$ radius, which would be inaccessible otherwise.

\section{Concept and Characterization}

Figure 1(a) and (b) show the concept of a 3D-printed AFM engine realized at the cleaved facet of a MCF (Fibercore, SM-7C). At the remote end, the MCF is coupled to a fiber-fanout (Fibercore, FAN-7C) connecting the seven cores with seven individual single-mode fibers (SMF). For opto-thermal actuation, the metal-covered cantilever is locally heated by a $785 \mathrm{~nm}$ laser, which is connected to the AFM engine through one of the fiber cores, see Fig. 1(a). The laser emission is periodically modulated close to the mechanical resonance frequency of the cantilever. The cantilever position is detected by measuring the optical back-reflection from a Fabry-Perot-type interferometer of length Lc, formed by the endface of a second fiber core and a concave freeform mirror at the bottom surface of the cantilever near the AFM tip. For read-out we use a tunable laser source (ECL, Santec, TSL-210) in combination with an optical circulator and a photodiode. We calibrate the position detector by a force-distance measurement [1] where the tip is brought into contact with and detached from a hard sample surface using a closed-loop piezoelectric positioner. The AFM engines were directly structured into a liquid acrylic negative-tone photoresist using a customized two-photon lithography system (Nanoscribe Photonic Professional GT, 40× objective lens with NA $=1.4$ ). We complemented the standard instrument with a proprietary high-precision automated alignment software for precise detection of the fiber core. Slicing and hatching writing distances of $100 \mathrm{~nm}$ were chosen for critical parts such as the mirror surface and the cantilever tip. To reduce the fabrication time, the cantilever itself was written with a $600 \mathrm{~nm}$ slicing distance. After lithography and development, a metal cover with layers of $5 \mathrm{~nm}$ chromium and $50 \mathrm{~nm}$ gold is deposited on the top surface of the structure using a highly directed electron-beam evaporation. 

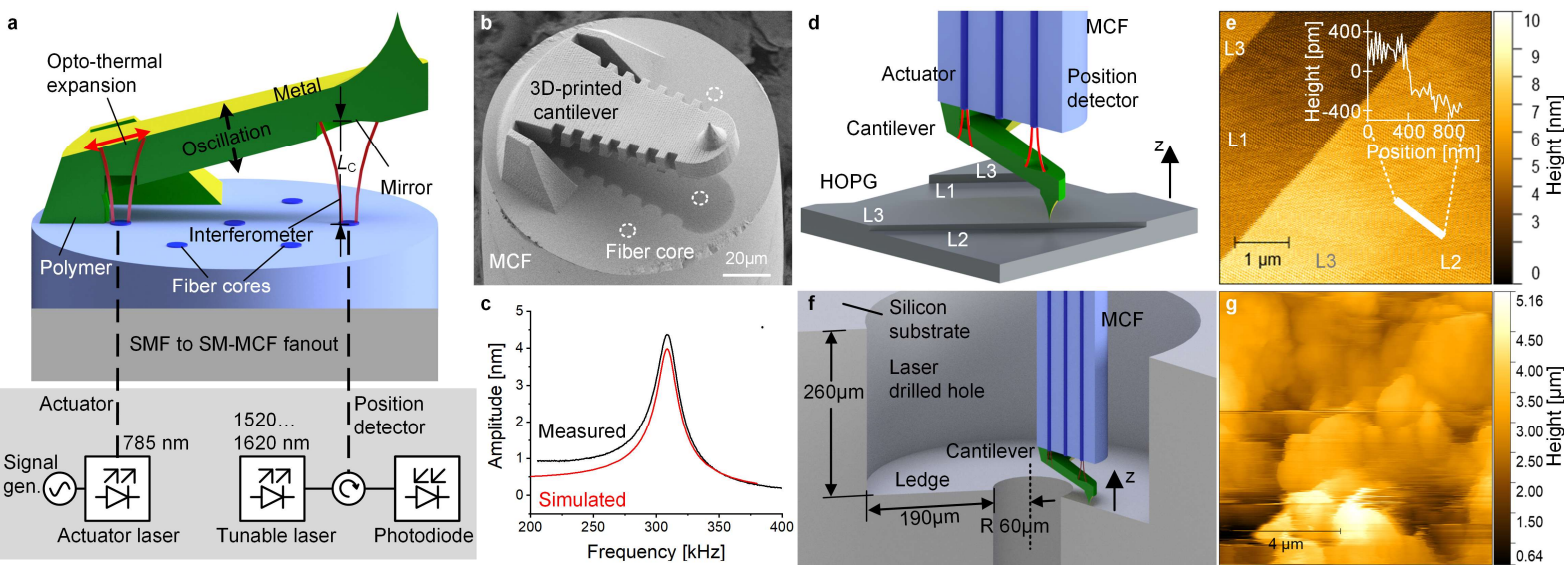

Figure 1: 3D-printed AFM engine on the facet of a single multi-core fiber (MCF). (a) Schematic view of the AFM engine (green) including a metal coating (yellow) and light propagation paths (red) on the facet of the MCF (blue). A fiber fan-out (not shown) is used to connect the MCF cores to individual single mode fibers (SMF). For opto-thermal actuation, the leftmost core is connected to a $785 \mathrm{~nm}$ laser that can be modulated in power. The rightmost core is used for position readout exploiting a Fabry-Perot-type interferometer formed by a freeform mirror and the endface of the fiber-core. (b) SEM image of a fabricated AFM engine. (c) Measured (black) and simulated (red) cantilever oscillation amplitude vs. actuation frequency. We find a resonance at approximately $308 \mathrm{kHz}$. (d) Schematic and (e) results of a topography measurement of highly oriented pyrolytic graphite (HOPG). The inset shows a line scan across a step of a single atomic layer. (f) Schematic and (g) results of a topography measurement on a ledge within a laser-drilled hole in silicon. The ledge exhibits a high surface roughness.

To characterize the dynamic behavior of the cantilever, we sweep the modulation frequency of the optical actuation power and measure the oscillation amplitude of the cantilever using the AFM position detector, see Fig. 1(c). The measured amplitude (black) is in good agreement with simulations (red, Comsol Multiphysics). This measurement was conducted with a commercial AFM controller (ARC2, Asylum Research Inc., Oxford Instruments) that comprises a signal generator and a lock-in amplifier for sensitive read-out of the position detector. For a freely oscillating cantilever, the maximum oscillation amplitude is $4.4 \mathrm{~nm}$ for a maximum actuation power of $1.5 \mathrm{~mW}$. The cantilever resonance frequency is $308 \mathrm{kHz}$. The $Q$-factor amounts to 11.1 which is sufficient for most measurements.

\section{Topography Measurements}

The lateral resolution of the AFM engine is determined by the shape of the tip, which has a curvature radius of $70 \mathrm{~nm}$. This radius can be further reduced to $25 \mathrm{~nm}$ by an appropriate choice of lithography parameters [4]. To demonstrate the vertical resolution, we measure the surface topography of a freshly cleaved highly oriented pyrolytic graphite (HOPG) sample, see Fig. 1(d,e). Three different layer heights L1, L2, L3 can be distinguished. A line scan indicated in white shows an atomic step height of $350 \mathrm{pm}$, which is in good agreement with published values of $335 \mathrm{pm}$ [1]. The ultra-small footprint of the AFM engine allows to investigate surface topographies within highaspect-ratio trenches or in deep holes. To demonstrate this capability, the MCF with the AFM engine was inserted into a laser-drilled hole in a silicon substrate for measuring the topography of the ledge within the hole, see Fig. 1(f,g). The extended part of the hole has a radius of $250 \mu \mathrm{m}$ and a depth of $260 \mu \mathrm{m}$. The measurement shows a high surface roughness with maximum height variations of $5 \mu \mathrm{m}$, Fig. $1(\mathrm{~g})$, caused by the heating and melting of the silicon substrate during laser drilling. In all measurements, scanning was accomplished by a lateral movement of the sample relative to the AFM engine. In general, our probe can access the bottom of holes with diameters as small as the fiber diameter $(125 \mu \mathrm{m})$ plus an appropriate clearance for moving the probe in the horizontal plane.

\section{Summary}

We demonstrate a fully functional ultra-compact AFM engine realized on the facet of a multi-core fiber. The AFM engine allows for high-precision dynamic-mode topography measurements and is perfectly suited for accessing narrow holes. To the best of our knowledge, this represents the most compact AFM probe head hitherto demonstrated.

This work was supported by the Deutsche Forschungsgemeinschaft (DFG, German Research Foundation) under Germany's Excellence Strategy via the Excellence Cluster 3D Matter Made to Order (EXC-2082/1 - 390761711), the BMBF project PRIMA, the Helmholtz International Research School for Teratronics (HIRST), the European Research Council (ERC Consolidator Grant 'TeraSHAPE', number 773248), the Alfried Krupp von Bohlen und Halbach Foundation, the NVIDIA Corporation, and the Karlsruhe NanoMicro Facility (KNMF) .

\section{References}

[1] P. Eaton and P. West, Atomic force microscopy (Oxford University Press, Oxford, 2010).

[2] D. Ianuzzi et al., Fibre-top cantilevers: design, fabrication and applications, Meas. Sci. Technol., 18, 3247-3252, (2007)

[3] P.-I. Dietrich et al., 3D-printed scanning-probe microscopes with integrated optical actuation and read-out, Small (in Print) (2019).

[4] G. Göring et al., Tailored probes for atomic force microscopy fabricated by two-photon polymerization, Appl.Phys.Lett., 109, 063101 (2016). 